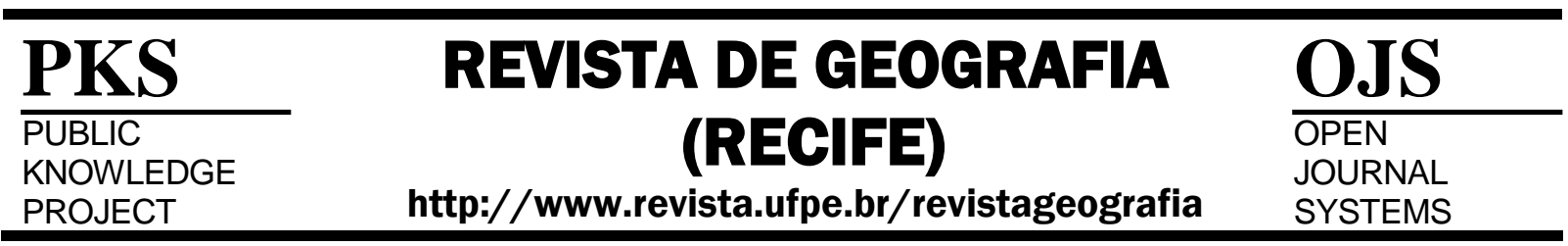

\section{CALIDAD DE AIRE EN BOGOTÁ: AUSENCIA DE INFORMACIÓN Y CAPTURA DE DATO EN EL 2015 POR MEDIO DE ANÁLISIS EN UN SISTEMA DE INFORMACIÓN GEOGRÁFICA}

\author{
Javier Enrique Aguilar Galindo \\ ${ }^{1}$ Geógrafo de la Universidad Nacional de Colombia. Investigador del Grupo de Investigación ESTEPA del \\ Departamento de Geografía de la Universidad Nacionald de Colombia; Email: querberoz@gmail.com
}

Artigo recebido em 30/01/2017 e aceito em 16/03/2017

\begin{abstract}
RESUMO
A qualidade do ar é uma questão que diz respeito aos vários atores estatais, porque mostram a poluição produzida e dispersos em uma área específica. Bogotá monitora contaminantes $\mathrm{NO}, \mathrm{NOx}, \mathrm{NO} 2, \mathrm{CO} 2, \mathrm{SO}$, PM10, PM2,5, CO e O3 através de Qualidade do Ar da Rede de Monitoramento e dados recolhidos a partir de 2015 Sistema de Informação gerados geograficamente, a fim de analisar a forma como estão concentrados na cidade usando para esta divisão em pequenas áreas. No processo, foi determinado que a captura de dados e a rede, não é eficiente, porque não é uma amostra comparável de energia entre as estações, de modo que apenas a concentração foi analisada em uma pequena área de Bogotá onde os dados são fiáveis . Embora o exercício permitiu a criação de um indicador que mede a concentração total de poluentes através dos índices cumulativos e adicioná-los a pequenas áreas, permitindo que áreas de estudo são as mais afetadas e o valor dos efeitos que você tem.
\end{abstract}

Palavras-chave: GIS, Poluentes, Ar, Mostrador, Kriging

\section{AIR QUALITY IN BOGOTA: ABSENCE OF INFORMATION AND DATA CAPTURE IN 2015 THROUGH ANALYSIS IN A GEOGRAPHIC INFORMATION SYSTEM}

\begin{abstract}
Air quality is an issue that concerns different state agents, because they show the rates of pollution that occur and are dispersed in a specific area. Bogotá carries out the monitoring of pollutants $\mathrm{NO}, \mathrm{NOX}, \mathrm{NO} 2, \mathrm{CO} 2, \mathrm{SO} 2$, PM10, PM2.5, CO and O3 through the Air Quality Monitoring Network and with the data collected from 2015 an Information System Geographical, in order to analyze how they are concentrated in the city using for this a division in small areas. In the process it was determined that the data capture, as well as the network, are not efficient, since there is no comparable sampling between stations, so only the concentration was analyzed in a small area of Bogotá where the data were reliable. Although the exercise allowed the creation of an indicator that measures the total concentration of contaminants by means of the accumulated indexes and add them to small areas, allowing to study the areas are most affected and the value of the affectation that you are.
\end{abstract}

Keywords: GIS, Contaminants, Air, Indicator, Kriging 


\section{INTRODUCCIÓN}

La calidad del aire dentro de las ciudades cobra importancia debido a los impactos en la salud que puede tener para sus pobladores. En ese marco, los gobernantes deben generar planes que permitan el control y mitigación, pero para ello se necesitan herramientas que permitan reconocer las zonas en donde se concentrar los contaminantes que se dispersan por el aire.

Para Colombia se cuenta con la resolución 0610 de 2010 en la cual se "establece la Norma de Calidad de Aire o Nivel de Inmisión, para todo el territorio nacional en condiciones de referencia" (2010) y que genera los protocolos para la medición de calidad de aire (comprendidos en dos tomos) usados en los diferentes departamentos y ciudades del territorio nacional. Sin embargo, dentro del proceso de organización de las dinámicas de medición no se cuenta con algún fragmento que mencione el proceso de análisis por unidades de área cartográficas, pues dependen en especial de la funcionalidad de las estaciones de toma de las muestras, así como el tratamiento de los datos.

De esa manera el objetivo de la investigación se basó en la evaluación de áreas con mayor cantidad de contaminantes que afectan la calidad del aire en la ciudad de Bogotá, comprendiendo que estos pueden diseminarse a través del aire con el efecto de las corrientes que se producen a lo largo del año (en este caso específico 2015) permitiendo una lectura cartográfica de las zonas con mayor afectación de la calidad de aire.

Por esta razón se trabajó con las bases de datos con las que cuenta el distrito capital

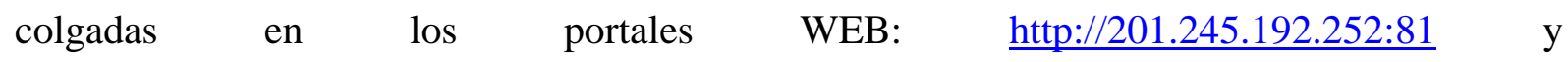
http://ambientebogota.gov.co/estaciones-rmcab las cuales pertenecen a la Secretaria de Ambiente de Bogotá. Allí se puede encontrar la ubicación de las estaciones de calidad de aire con las cuales cuenta la ciudad por medio de la Red de Monitoreo de Calidad de Aire para el Distrito Capital.

Es así como en un primer momento se explicaran brevemente los contaminantes que se miden en la ciudad de Bogotá a través de la red de calidad de aire, seguido del proceso metodológico y las fuente de información con la cual se trabajó, para continuar con el análisis de la información en el Sistema de Información Geográfico sobre Calidad de Aire en Bogotá y se terminan con el análisis geoespacial de los hallazgos encontrados junto con algunas recomendaciones. 


\section{ALGUNOS ESTUDIOS SOBRE LA IMPORTANCIA DE LA CALIDAD DE AIRE}

La calidad de aire en las zonas urbanas tienen efectos en la población, y esto constituye la toma de decisiones claras para mitigar, controlar o evitar la dispersión de estos contaminantes. Varios autores (CAÑADA TORRENCILLA e MORENO JIMÉNEZ, 2015) (ROMERO e OPAZO, 2011) (BELTRÁN CALVO, 2013) hacen referencia a la justicia ambiental como eje articulador para comprender como se adquiere: la información, la ausencia de toma de decisiones y la afectación en la población.

Sin embargo, el tema de la calidad de aire a avanzado y la tecnologías pueden apoyar estos procesos, teniendo en cuenta el uso de los Sistemas de Información Geográfica (QUICO BERRIO, 2012), a través de procesos de interpolación (ASTUDILLO ROMERO, 2012) (COLSA PÉREZA, GONZÁLEZ LORENZOB, et al., 2014) (CAÑADA TORRECILLA, MORENO JIMÉNEZ e GONZÁLEZ LORENZO, 2014) (RODRÍGUEZ RODRÍGUEZ, 2014) (GARCÍA ÁVILA e ROJAR, 2016). Posada (POSADA PARRA, 2015) realiza un aplicativo web para acceder a la información sobre contaminación del aire, lo que permite conocer en tiempo real el comportamiento de la calidad de aire, este proceso se une a la forma de visualización de la información en cartografía.

Los autores no hacen referencia a un análisis integral de la información de los contaminantes que se producen, debido a que estos son diferentes, por lo tanto analizan la concentración que cada uno de estos tiene, pues permite comprender cuál de estos es el que tiene mayor grado de impacto para la población (CAÑADA TORRENCILLA e MORENO JIMÉNEZ, 2015) (COLSA PÉREZA, GONZÁLEZ LORENZOB, et al., 2014) (RODRÍGUEZ RODRÍGUEZ, 2014) (ASTUDILLO ROMERO, 2012) (FERNÁNDEZ GARCÍA, 2005)

Por otra parte, los estudios sobre la calidad de aire permiten encontrar las fuentes de las cuales se producen estos contaminantes, en los cuales se cuentan los fijos o móviles (GALARZA RODRIGO, 2010) (LONDOÑO, CORREA e PALACIO, 2011). Por lo tanto, si se encuentran aquellos elementos que producen la contaminación se puede generar unas políticas que permitan establecer el control de las mismas.

Además, existen otros métodos para el análisis espacial de la calidad de aire, entre ellos se encuentra el uso de bioindicadores, en especial líquenes que son especialmente sensibles a los contaminantes, permitiendo su estudio por la aparición o ausencia de los 
mismos (ELJAUDE, 1995) (HAWKSWORTH, ITURRIAGA e CRESPO, 2005) (MENA, 2012).

Desde otras perspectivas del análisis de la contaminación de aire puede verse el efecto que tienen estos contaminantes para la población. Beltrán (2013) realiza un análisis de los contaminantes de aire en la salud de la población de Bogotá, en especial con las enfermedades respiratorias y que para el presente caso puede ayudar a entender porque su análisis se centró sobre un área específica de la ciudad.

El tema de calidad de aire tiene relevancia, pues afecta de diferentes formas a las poblaciones y es en esa impronta que los diferentes autores que he mencionado muestran la importancia de espacializar este fenómeno de contaminación. Encontrar las áreas, o el contaminante con mayor dispersión son esenciales para todos ellos y ellas, pues se puede ayudar a tomar decisiones, lo cual pone todos estos ejercicios a disposición de los gobernantes para que puedan utilizarlos en la toma de decisiones y así mitigar efectos nocivos para las personas.

Sin embargo, considero que estos estudios basados en la construcción de modelamiento a partir de un sistema de información geográfica tienen variabilidades que pueden complementarse, comprendiendo que se dan concentraciones en diferentes áreas, por ello, la importancia radica en la construcción de una herramienta que analice las áreas de mayor impacto.

\section{CONTAMINANTES}

Lo primero que se debe tener en cuenta es la necesidad de comprensión que deben tener los diferentes contaminantes en la salud humana, en ese sentido Fernández nos explica como "la calidad del aire y la confortabilidad climática son dos aspectos esenciales para definir la calidad desde la óptica ambiental, debido a sus efectos negativos sobre la salud" (2005, p. 508). Los cuales, entendiendo las dinámicas de las corrientes de aire afectan en mayor o menor medida ciertas áreas debido a los contaminantes que en estas zonas se producen.

Segú la normatividad colombiana se entiende como contaminantes:

“fenómenos físicos o sustancias, o elementos en estado sólido, liquido o gaseoso, causantes de efectos adversos en el medio ambiente, los recursos naturales renovables 
y la salud humana que, solos o en combinación, o como producto de reacción, se emiten al aire como resultado de actividades humanas, de causas naturales, o de una combinación de estas" (MINISTERIO DE AMBIENTE, 2010)

La Secretaría de Ambiente de Bogotá adquiere información de los siguientes contaminantes:

a. "SO2: El óxido de azufre (IV) o dióxido de azufre cuya fórmula es SO2 es un gas incoloro con un característico olor asfixiante. Se trata de una sustancia reductora que con el tiempo y en contacto con el aire y la humedad se convierte en trióxido de azufre. [...].

b. PM10: Se denomina PM10 a pequeñas partículas sólidas o líquidas de polvo, cenizas, hollín, partículas metálicas, cemento o polen, dispersas en la atmósfera, y cuyo diámetro varía entre 2,5 y 10 нm (1 micrómetro corresponde la milésima parte de 1 milímetro). Están formadas principalmente por compuestos inorgánicos como silicatos y aluminatos, metales pesados entre otros, y material orgánico asociado a partículas de carbono (hollín). Las PM10 al ser inhaladas y al penetrar con facilidad al sistema respiratorio humano, causan [...].

c. PM2.5: [...] representa partículas de menos de 2,5 $\mu \mathrm{m}$ de diámetro aerodinámico [...] La generación de aerosoles puede ser de origen natural o debido a la actividad humana. Algunas partículas se dan de manera natural, procedentes de los volcanes, las tormentas de polvo, los incendios forestales y de pastizales, y la pulverización de agua marina. Las actividades humanas, como la quema de combustibles y la alteración de la superficie terrestre también generan aerosoles [...].

d. CO: El monóxido de carbono u óxido de carbono (II) cuya fórmula química es CO, es un gas inodoro, incoloro, inflamable y altamente tóxico. Puede causar la muerte cuando se respira en niveles elevados. Se produce cuando se queman materiales combustibles como gas, gasolina, keroseno, carbón, petróleo, tabaco o madera en ambientes de poco oxígeno. [...]

e. O3: El ozono es un gas altamente reactivo de color azul pálido, constituido por tres átomos de oxígeno en su estructura molecular. El ozono se considera como uno de los contaminantes de mayor preocupación en la actualidad, ya que es altamente oxidante y afecta a los tejidos vivos, se asocia con diversos padecimientos en la salud humana. $[\cdots]$. 
f. NO2: El óxido de nitrógeno (IV) o dióxido de nitrógeno (NO2), es un compuesto químico formado por los elementos nitrógeno y oxígeno, uno de los principales contaminantes entre los varios óxidos de nitrógeno. [...] Se forma como subproducto en los procesos de combustión a altas temperaturas, como en los vehículos motorizados y las plantas eléctricas" (SECRETARÍA DE AMBIENTE DE BOGOTÁ, 2016).

Como menciona Londoño, Correa y Palacio (2011) la medición de estos contaminantes atmosféricos es importante y dan un orden a las actividades políticas que se establecen dentro de los sectores para mitigar, controlar y vigilar dichas emisiones. Elementos que se debe tener en cuenta para la formulación en acción tangibles, pero en necesario que se analice las emisiones que se han dado en Bogotá, con los cuales se puede encontrar los vacíos en la adquisición del dato, lo que da un primer acercamiento a la ausencia de verdaderas lecturas sobre el comportamiento de los contaminantes.

"El mecanismo que garantiza la efectividad del proceso y la calidad de la información es el Plan de Calidad del SVCA, el cual deberá desarrollarse a lo largo de todo el proceso a fin de garantizar la confiabilidad de la información reportada. Dentro de este plan se incluyen las actividades de mantenimiento y calibración de equipos, que garantizan su operatividad y la veracidad de las lecturas y registros de las muestras tomadas” (MINISTERIO DE AMBIENTE, 2010, p. 12)

\section{FUENTES DE INFORMACIÓN Y METODOLOGÍA}

Las fuentes de información correspondiente se desprenden de la Red de Monitoreo y Calidad de Aire de la Secretaria de Ambiente de Bogotá. Los cuales se pueden visualizar a través de página web, en el portal se puede descargar las tablas con los datos reportados por las estaciones. La ubicación de las estaciones se muestran en el mapa uno, donde se puede ver la falta de estación para la localidad de Usme, Sumapaz, Ciudad Bolívar y Bosa. 

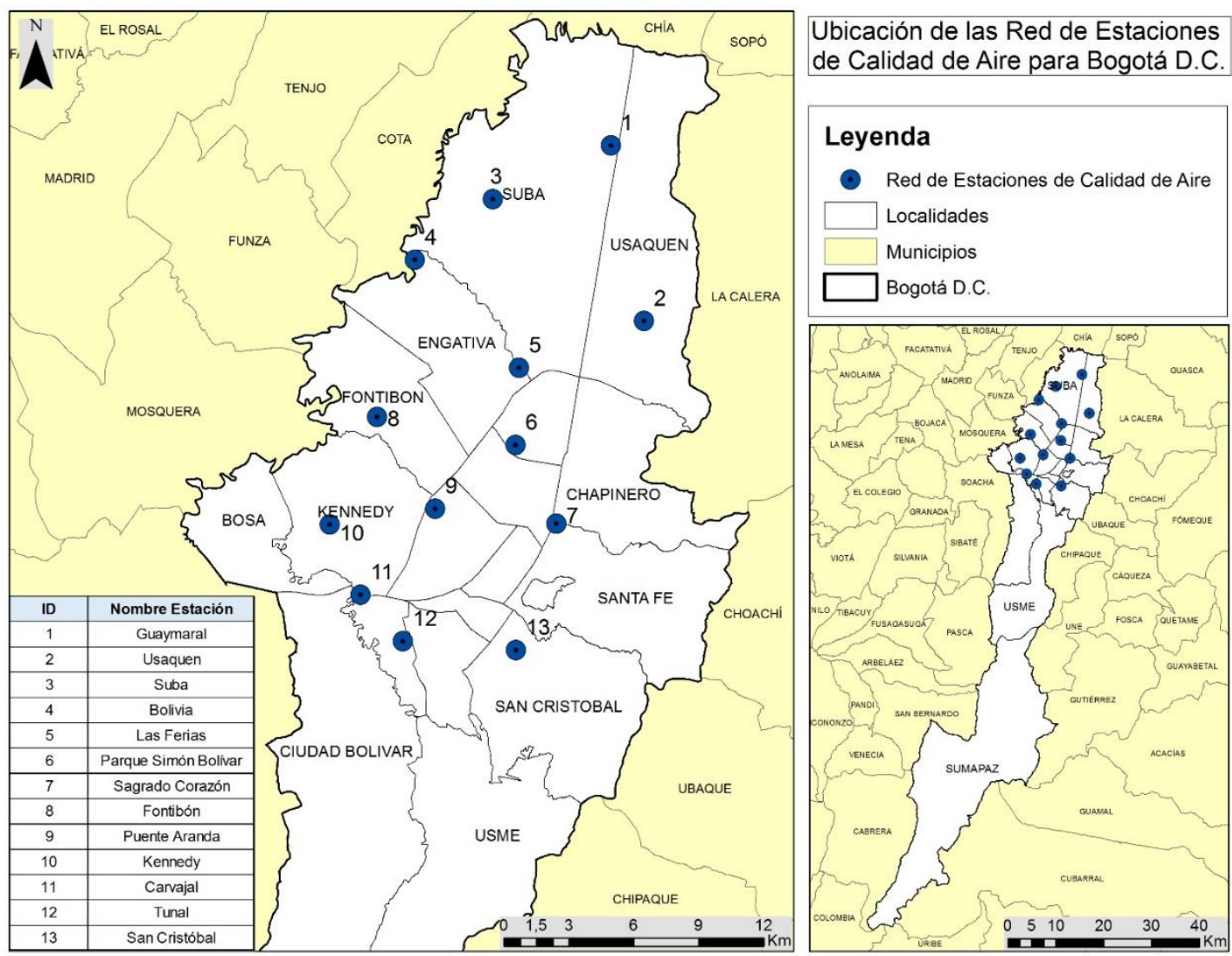

Mapa 1.Ubicación de Estaciones Red de Monitoreo de Calidad de Aire del Distrito Capital de Bogotá. Fuentes: Secretaria de Ambiente e IDECA. Elaboración Propia.

En la tabla uno se puede ver los valores medios anuales para los diferentes contaminantes captados por la red, aunque el único contaminante que se pudo captar en todas las estaciones es el correspondiente al PM10, mientras que las estaciones que miden menos contaminantes de calidad de aire son Sagrado Corazón en donde no se captan cinco de las ocho muestras, Usaquén solo mide cuatro de ocho al igual que Suba y San Cristóbal solo mide tres de ocho. Las únicas que miden todos los elementos contaminantes (junto con dirección del viento y la velocidad del mismo) son Carvajal, Parque Simón Bolívar y Las Ferias.

\begin{tabular}{|c|c|c|c|c|c|c|c|c|c|c|}
\hline Estación & $\begin{array}{c}\text { Dirección } \\
\text { Viento } \\
(\text { Grados })\end{array}$ & $\begin{array}{c}\text { Velocidad } \\
\text { Viento } \\
(\mathrm{m} / \mathrm{s})\end{array}$ & $\mathrm{CO}(\mathrm{ppm})$ & $\mathrm{NO}(\mathrm{ppb})$ & $\begin{array}{c}\text { NO2 } \\
(\mathrm{ppb})\end{array}$ & $\begin{array}{c}\text { NOX } \\
(\mathrm{ppb})\end{array}$ & $\begin{array}{c}\text { OZONO } \\
(\mathrm{ppb})\end{array}$ & $\begin{array}{c}\text { PM10 } \\
(\mu \mathrm{g} / \mathrm{m} 3)\end{array}$ & $\begin{array}{c}\text { PM2.5 } \\
(\mu \mathrm{g} / \mathrm{m} 3)\end{array}$ & $\begin{array}{c}\text { SO2 } \\
(\mathrm{ppb})\end{array}$ \\
\hline Carvajal & 183,34 & 2,54 & 1,20 & 66,69 & 27,99 & 94,57 & 7,76 & 86,25 & 30,39 & 4,27 \\
\hline $\begin{array}{c}\text { Parque Simón } \\
\text { Bolívar }\end{array}$ & 177,97 & 1,43 & 0,61 & 21,51 & 17,28 & 38,71 & 13,14 & 28,01 & 16,89 & 1,28 \\
\hline Guaymaral & 138,33 & 1,02 & Sin dato & 22,70 & 12,92 & 35,55 & 11,30 & 30,73 & 13,61 & Sin dato \\
\hline
\end{tabular}




\begin{tabular}{|c|c|c|c|c|c|c|c|c|c|c|}
\hline Kennedy & 171,22 & 2,73 & 0,63 & 28,61 & 13,53 & 42,14 & Sin dato & 65,97 & 26,81 & 2,03 \\
\hline Las Ferias & 125,60 & 1,90 & 0,54 & 15,09 & 22,03 & 37,41 & 16,68 & 34,59 & 16,57 & 1,25 \\
\hline Sagrado Corazón & 138,66 & 1,70 & Sin dato & Sin dato & Sin dato & Sin dato & 11,35 & 33,31 & 14,30 & Sin dato \\
\hline Puente Aranda & 183,22 & 2,30 & 0,71 & 22,48 & 17,13 & 39,73 & 8,76 & 51,98 & Sin dato & 1,19 \\
\hline San Cristóbal & 118,83 & 1,17 & 0,46 & Sin dato & Sin dato & Sin dato & 15,38 & 25,22 & 8,46 & 0,39 \\
\hline Suba & 111,45 & 1,82 & Sin dato & Sin dato & Sin dato & Sin dato & 10,80 & 47,20 & 21,35 & 3,16 \\
\hline Tunal & 188,70 & 1,18 & 0,61 & 15,62 & 15,11 & 30,76 & 10,03 & 42,34 & 21,04 & Sin dato \\
\hline Usaquén & 163,14 & Sin dato & 0,39 & Sin dato & Sin dato & Sin dato & 23,78 & 29,44 & 12,58 & Sin dato \\
\hline
\end{tabular}

Tabla 1. Promedios anuales correspondientes a los contaminantes atmosféricos.

Aunque se cuenta con los promedios anuales es necesario aclarar varias cosas sobre estos antes de continuar.

1. No se completaron los datos para los meses faltantes, debido a que estos pueden modificar el proceso de análisis de contaminantes, siendo necesario explicar porque el uso de esos datos imaginarios.

2. Los datos, en relación a los promedios anuales, no muestran los picos más altos, los cuales pueden ser producidos por diferentes elementos en la ciudad, como por ejemplo los días sin carro, el incendio de una empresa de juegos pirotécnicos y otros eventos que disminuyen o aumentan los gases contaminantes que se esparcen por el aire.

3. Si bien las corrientes del aire son más o menos constantes hay meses en los cuales estos pueden cambiar radicalmente de dirección, modificando como se esparcen los contaminantes.

Con esos datos se procedió a realizar un Sistema de Información Geográfica, en el cual se realizaron varios geoprocesamientos: primero se procedió a georeferenciar las estaciones, y junto a estas los diferentes valores de los contaminantes, con el objetivo de pasar esta información a raster ${ }^{1}$, encontrando de esta manera la dispersión que tienen los datos entre cada uno por medio de la herramienta kriging (COLSA PÉREZA, GONZÁLEZ LORENZOB, et al., 2014), el cual permite analizar la distribución entre puntos con un grado de información, permitiendo encontrar relaciones entre estos (CAÑADA TORRECILLA, MORENO JIMÉNEZ e GONZÁLEZ LORENZO, 2014), o en otras palabras, “es un método o conjunto de métodos de interpolación espacial, que se basa en predicciones realizadas a través del error medio cuadrático" (RODRÍGUEZ RODRÍGUEZ, 2014, p. 13). El software (ArcGIS) utiliza esta información y la procesa en varios cortes, como el proceso de

${ }^{1}$ La información raster se considera continua, debido a que su representación se realiza en pixel con información binaria la cual es representada en una malla continua. 
contaminantes de aire se aproxima a procesos de distribución en relación uno a uno se escogió el método Gaussian. En la imagen 1 se muestra el procesamiento matemático que se realiza, donde se representa la curva generada a partir de la dispersión de los datos, los cuales se concentran en áreas, permitiendo encontrar relaciones continuas entre los datos.

\section{GAUSSIAN}

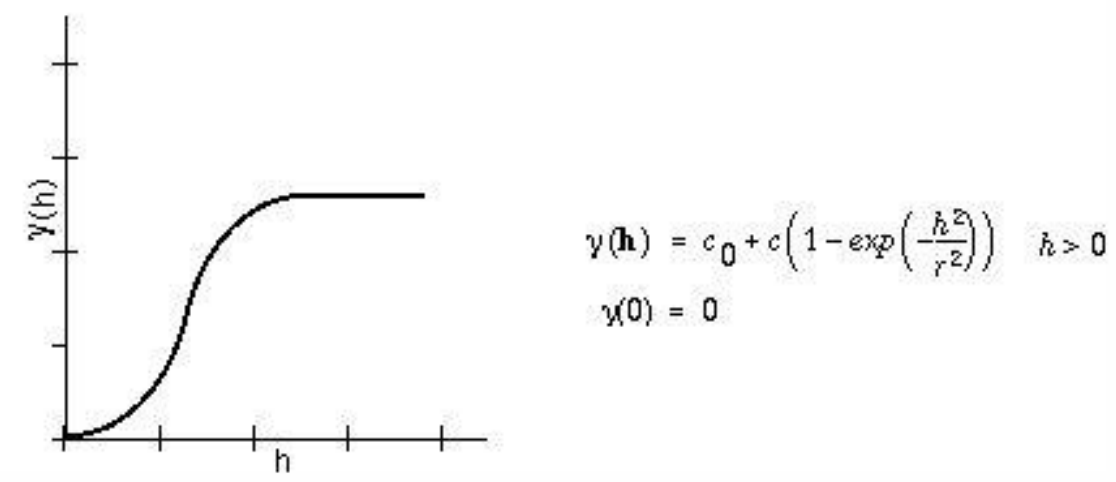

Ilustración de modelo de semivarianza gaussiana

Imagen 1. Modelo Gaussiano utilizado por ArcGIS en la realización del Kriging. Tomado de https://pro.arcgis.com/es/pro-app/tool-reference/3d-analyst/how-krigingworks.htm\#ESRI_SECTION1_DD457E27190A4CD0BC1D7A9D7BA5DD5F

El modelo basado en la función gaussiana es el más utilizado en casos puntuales ${ }^{2}$, aunque existen los modelos globales, o regionales-locales que pueden ser utilizados de manera más eficaz, pues permiten la correlación de variables y entienden la estructura del espacio atmosférico (MOSCOSO VANEGAS, ASTUDILLO ALEMÁN e VÁZQUEZ FREIRE, 2015), pero en este caso, al tener información de manera escasa y sobre algunas estaciones, es necesario interpolarlas con esa función.

Aquellos que permiten adquirir la información continua y pasarla a discreta son los que mejor se ajustan a los procesos de análisis cartesiano, con lo que se permite mejorar la correlación de las diferentes variables a tener en cuenta, lo cual hace necesario que el planteamiento generado por el método Gaussian en el software permita encontrar las áreas en dicha dispersión. Los resultados de este primer paso se pueden observar en el mapa dos y tres, en donde se ven los resultados para cada uno de los contaminantes, así como una clasificación natural para poder ver los cortes de los datos.

${ }^{2}$ Haciendo referencia a los shapes punto. Aguilar Galindo, 2017 ISSN 0104-5490 177 

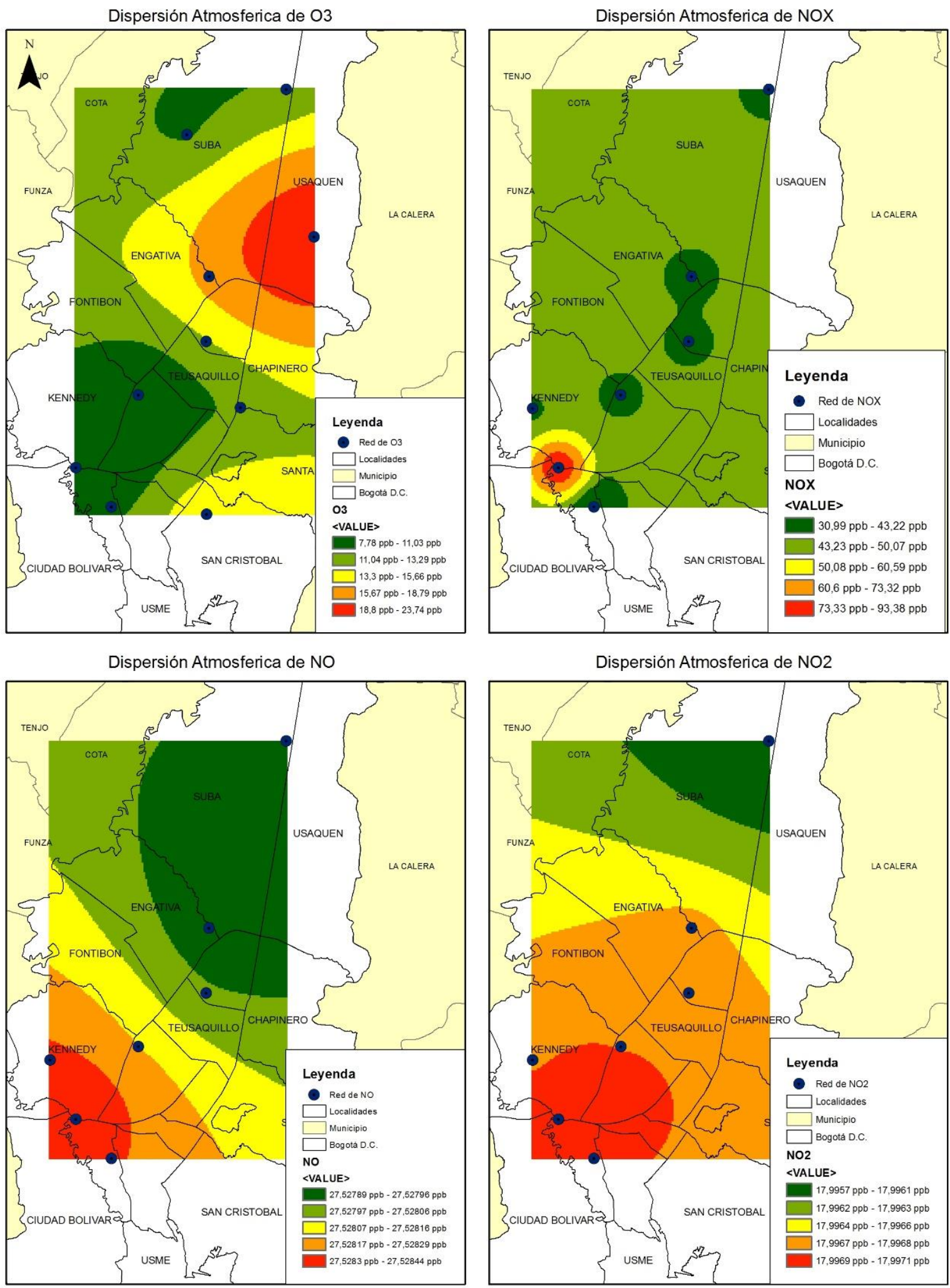

Mapa 2. Cartografía de la dispersión de contaminantes O3, NOX, NO, NO2 a partir de la herramienta Kriging. Datos de la Red de Calidad de Aires, IDECA. Elaboración propia. 

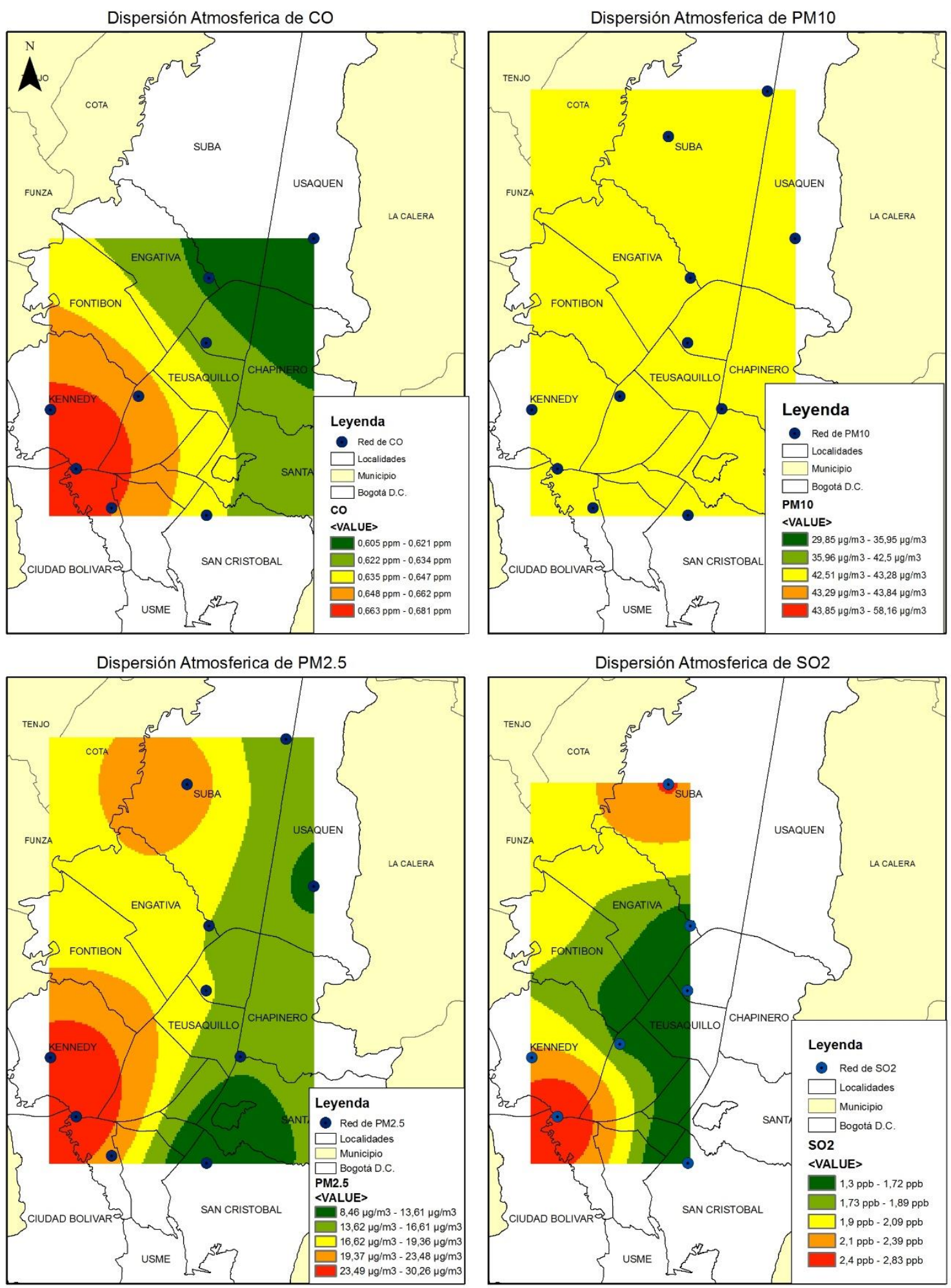

Mapa 3. Cartografía de la dispersión de contaminantes CO, PM10, PM2.5 y SO2 a partir de la herramienta Kriging. Datos de la Red de Calidad de Aire, IDECA. Elaboración propia. 
Sin embargo, como menciona Cañada, Moreno y González, en la interpolación pueden presentarse los siguientes problemas: "Datos escasos y con representatividad espacial limitada [...], las técnicas de interpolación espacial pueden considerar a la vez herramientas simples y sofisticadas [...], las estimaciones espaciales son de difícil validación, salvo en los escasos puntos muéstrales [...],los procesos generadores de patrones espaciales son complejos y, además, los mecanismos operando en cada tipo de proceso presentan bastantes especificidades" (2014, p. 319-320).

Luego de este proceso de interpolación (y comprendiendo las dificultades al traspasar la información a un formato raster) se generó un fishnet, el cual genera dos mallas: una de puntos y otra de polígonos (cuadrados de 250X250), a este último shape $e^{3}$ se adiciono la información de todos los contaminantes por medio de un análisis estadístico dentro del software, con el objetivo de encontrar los valores medios asociados a cada una las áreas generadas, utilizando la herramienta zonal statistics as table.

Una vez realizado ese proceso se encuentran los índices de calidad de aires para el NO2, SO2, O3, CO, PM10 y PM2.5 (se excluyen NO y NOX) ${ }^{4}$. Con los cuales se procede a generar el indicador de calidad de área. La ecuación utilizada corresponde a:

$$
\mathrm{INCA}=\frac{\left[\sum \mathrm{ICA}=\left(\left(I_{H I}-I_{L O} / B P_{H I}-B P_{L O}\right) *\left(C_{I}-B P_{L O}\right)\right)+I_{L O}\right] * 100}{500}
$$

INCA: Indicador de Contaminación por Área.

ICA: Índice de Calidad de Aire ${ }^{5}$

$I_{H I}$ : Valor del índice en el límite superior en la categoría del ICA

$I_{L O}:$ Valor del índice en el límite inferior en la categoría del ICA

$B P_{H I}$ : Punto de quiebre de la concentración en el límite superior de la categoría del ICA

$B P_{L O}$ : Punto de quiebre de la concentración en el límite inferior de la categoría del ICA

$C_{I}$ : Concentración del contaminante

La tabla dos muestra los valores para la elaboración de los procedimientos matemáticos correspondientes a los índices de calidad de aire. Cabe señalar que la

\footnotetext{
${ }^{3}$ El Shape es una capa vectorial en tres tipos de forma: punto, línea y polígono.

${ }^{4}$ Debido a que no se cuenta con una medida variable para el Monóxido de Nitrógeno y Otro Nitrógenos (pues algunas estaciones pueden medir el NO y NOX como NO2) se eximen del procedimiento para que no se generen errores en la detección de las áreas.

${ }^{5}$ La ecuación de los índices correspondientes es tomada de http://www.metropol.gov.co/CalidadAire/Paginas/ica.aspx 
clasificación de color que allí se muestra se realiza con el fin de poder informar a la población sobre la concentración del contaminante, ya que cualquiera de estos puede causar la muerte si la exposición es continua, aunque en los niveles marrones se considera el grado máximo de exposición, lo cual acarrearía la muerte de la persona que se encuentra expuesta.

\begin{tabular}{|c|c|c|c|c|c|c|c|c|c|}
\hline ICA & Color & Clasificación & $\begin{array}{c}\mathrm{O} 3(8 \mathrm{~h} \\
\mathrm{ppm})\end{array}$ & $\begin{array}{c}\mathrm{O} 38(1 \mathrm{~h} \\
\mathrm{ppm})\end{array}$ & $\begin{array}{c}\text { PM10 } \\
(\mu \mathrm{g} / \mathrm{M} 3)\end{array}$ & $\begin{array}{l}\text { PM2.5 } \\
(\mu \mathrm{g} / \mathrm{M} 3)\end{array}$ & $\mathrm{CO}(\mathrm{ppm})$ & $\mathrm{SO} 2$ (ppm) & NO2 (ppm) \\
\hline \multirow{2}{*}{$0-50$} & \multirow{2}{*}{ Verde } & \multirow{2}{*}{ Buena } & 0 & - & 0 & 0 & 0 & 0 & 0 \\
\hline & & & 0,059 & - & 54 & 12 & 4,4 & 0,035 & 0,053 \\
\hline \multirow{2}{*}{$51-100$} & \multirow{2}{*}{ Amarillo } & \multirow{2}{*}{ Moderada } & 0,06 & - & 55 & 12,1 & 4,5 & 0,036 & 0,054 \\
\hline & & & 0,075 & - & 154 & 35,4 & 9,4 & 0,075 & 0,1 \\
\hline \multirow{2}{*}{$101-150$} & \multirow{2}{*}{ Naranja } & \multirow{2}{*}{$\begin{array}{c}\text { Dañina para } \\
\text { grupos sensibles }\end{array}$} & 0,076 & 0,125 & 155 & 35,5 & 9,5 & 0,076 & 0,101 \\
\hline & & & 0,095 & 0,164 & 254 & 55,4 & 12,4 & 0,185 & 0,36 \\
\hline \multirow{2}{*}{$151-200$} & \multirow{2}{*}{ Rojo } & \multirow{2}{*}{$\begin{array}{c}\text { Dañina para la } \\
\text { salud }\end{array}$} & 0,096 & 0,165 & 255 & 55,5 & 12,5 & 0,186 & 0,361 \\
\hline & & & 0,115 & 0,204 & 354 & 150,4 & 15,4 & 0,304 & 0,649 \\
\hline \multirow{2}{*}{$201-300$} & \multirow{2}{*}{ Púrpura } & \multirow{2}{*}{$\begin{array}{l}\text { Muy Dañina a la } \\
\text { salud }\end{array}$} & 0,116 & 0,205 & 355 & 150,5 & 15,5 & 0,305 & 0,65 \\
\hline & & & 0,374 & 0,404 & 424 & 250,4 & 30,4 & 0,604 & 1,249 \\
\hline \multirow{2}{*}{$301-500$} & \multirow{2}{*}{ Marrón } & \multirow{2}{*}{ Peligrosa } & - & 0,405 & 425 & 250,5 & 30,5 & 0,605 & 1,25 \\
\hline & & & - & 0,604 & 604 & 500,4 & 50,4 & 1,004 & 2,049 \\
\hline
\end{tabular}

Tabla 2. Puntos de Corte del ICA. Modificada de

http://www.metropol.gov.co/CalidadAire/Paginas/ica.aspx

En el proceso para encontrar cada uno de los ICA se determinó que para los promedios anuales solamente el PM2.2 se encuentra en nivel amarillo, los demás contaminantes están en el nivel bajo para cada una de las áreas construidas, diferente a lo toma puntual generada por las estaciones de la red de monitoreo de calidad de aire. La necesidad de generar el indicador nace de ese proceso de dispersión por área, con lo cual se revelará las zonas de mayor concentración del contaminante a partir de las medidas medias de cada uno de los polígonos desarrollados en el fishnet.

\section{RESULTADOS Y ANÁLISIS}

Al realizar los diferentes geoprocesamientos se encontró que existe solo una pequeña zona en la cual los datos de los diferentes contaminantes pueden tomarse como ciertos, en la intersección de los diferentes raster. Esta área es la zona con datos completos, y sobre esta se puede hacer el análisis correspondiente de afectación por el INCA. Aunque, debido a la naturaleza de los procesamientos cartográficos que se mostrarán a continuación se agrupo la 
información en cinco indicadores textuales. La tabla tres muestra la relación numérica del INCA que se aplicó en el análisis espacial.

\begin{tabular}{|l|c|c|c|c|}
\hline \multicolumn{5}{|c|}{ INCA ZONA CON DATOS COMPLETOS } \\
\hline INCA & Valor mayor & Valor mínimo & Valor promedio & $\begin{array}{c}\text { Área total } \\
\text { afectada (KM2) }\end{array}$ \\
\hline ALTO & 32,17 & 27,55 & 29,86 & 23,44 \\
\hline MEDIO ALTO & 27,55 & 25,19 & 26,37 & 64,69 \\
\hline MEDIO & 25,19 & 23,53 & 24,36 & 12,31 \\
\hline MEDIO BAJO & 23,53 & 21,25 & 22,39 & 4,56 \\
\hline BAJO & 21,25 & 16,90 & 19,08 & 0 \\
\hline
\end{tabular}

Tabla 3. Valores del INCA asociados a la representación cartográfica.

En la zona de datos completos el INCA Bajo no aparece área afectada, a pesar que si hay una franja de estos datos sobre la zona oriental de Bogotá, pero se desestiman debido a que no se cuenta con el total de ICA, lo que fracciona la labor estudiada a los cuatro

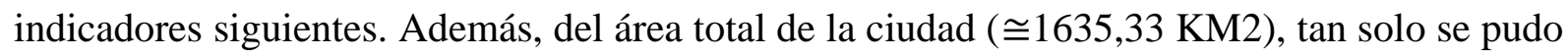
estudiar $105 \mathrm{KM} 2$, lo cual hace referencia al 6,42\% de la ciudad, un porcentaje muy bajo que lastimosamente no puede mostrar la dinámica de la calidad de aire en Bogotá. Para los INCA Medio Bajo, Medio no tienen un área de afectación muy grande a diferencia de Medio Alto quien tiene el área más grande de afectación y que junto con Alto ocupan el 83,9\% del área total estudiada, la cual puede verse en el mapa cuatro. En este se puede ver la relación existente entre el área total del fishnet generado con todos los datos, así como el área definitiva con la cual se realizan los análisis.

Dentro de la zona de datos completos se encuentran ubicada una estación en la cual no se muestrea los contaminantes de Calidad de Aire, allí se toma el dato correspondiente a dirección y velocidad de viento (ubicada en la localidad de Fontibón), a pesar de ello esta pertenece a la Red de Monitoreo de Calidad de Aire, por eso la presencia dentro del mapa (al igual que la estación de Bolivia que tiene esta misma característica). Si la estación estuviera operando en su totalidad seguramente se tendrían variaciones asignadas dentro de la zona demarcada, siendo menor o mayor el valor del INCA (dependiendo de los muestreos que se pudieran adquirir). 


\section{Calidad de Aire por Áreas en Bogotá}

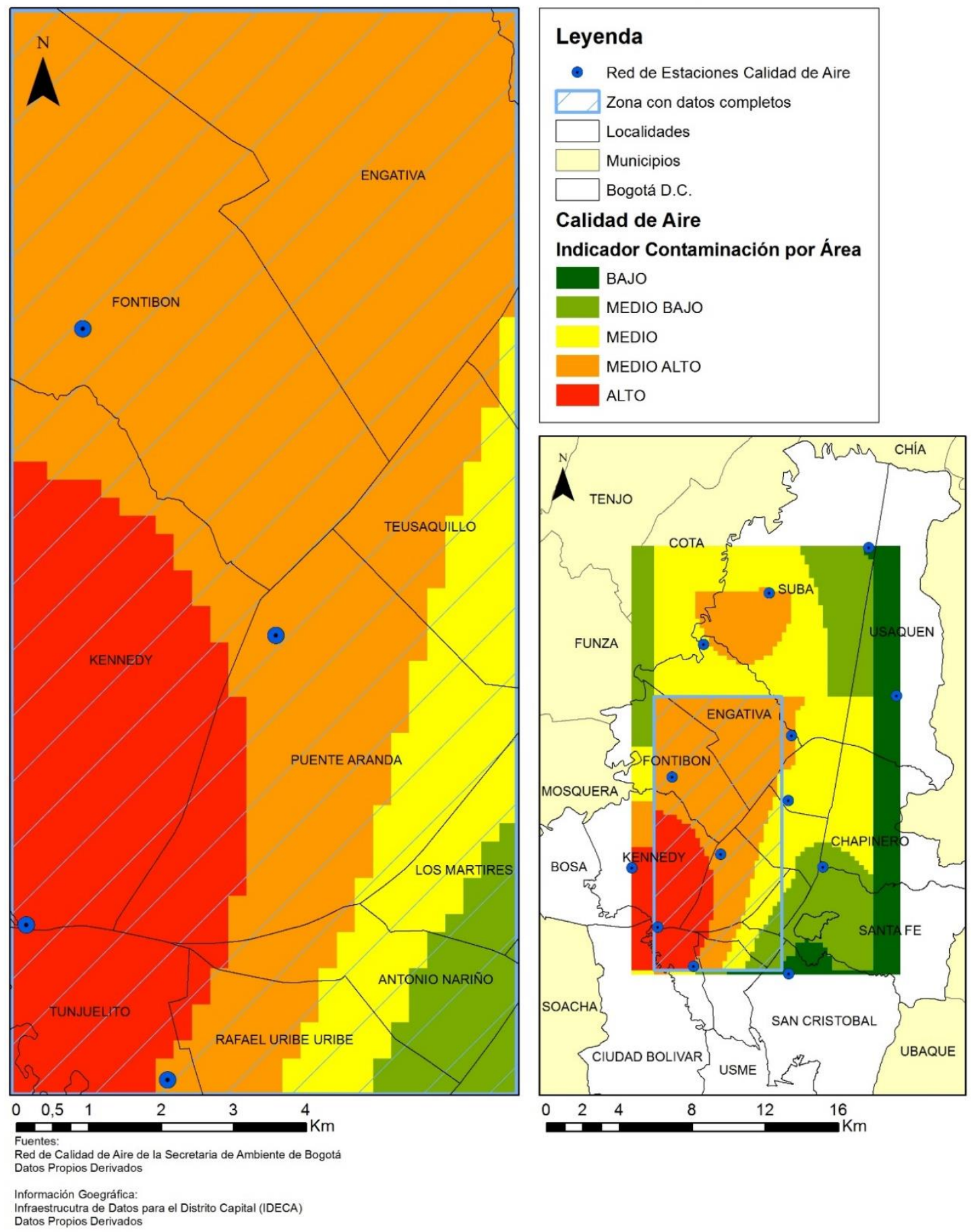

Mapa 4. Calidad de aire por área para Bogotá. Elaboración propia.

La ciudad cuenta con una división administrativa de 20 localidades, y dentro de la zona de datos completos se realizó análisis parcial de 14 localidades (ninguna en un 100\%), la 
tabla 4 muestra los resultados del total del área afectada según el tipo de INCA presente dentro de su perímetro ${ }^{6}$.

\begin{tabular}{|c|c|c|c|c|}
\hline ID & Localidad & INCA & $\begin{array}{l}\text { Área afectada } \\
\text { (KM2) }\end{array}$ & $\begin{array}{c}\text { Porcentaje de área afectada por } \\
\text { localidad }\end{array}$ \\
\hline \multirow{4}{*}{1} & \multirow{4}{*}{ Antonio Nariño } & ALTO & 0,144 & \multirow{4}{*}{98,4} \\
\hline & & MEDIO & 1,316 & \\
\hline & & MEDIO ALTO & 1,076 & \\
\hline & & MEDIO BAJO & 2,260 & \\
\hline \multirow{2}{*}{2} & \multirow{2}{*}{ Barrios Unidos } & MEDIO & 0,338 & \multirow{2}{*}{5,2} \\
\hline & & MEDIO ALTO & 0,284 & \\
\hline 3 & Ciudad Bolívar & ALTO & 0,295 & 0,2 \\
\hline \multirow{2}{*}{4} & \multirow{2}{*}{ Engativá } & MEDIO & 0,000 & \multirow{2}{*}{57,7} \\
\hline & & MEDIO ALTO & 20,670 & \\
\hline 5 & Fontibón & MEDIO ALTO & 22,665 & 68,2 \\
\hline \multirow{2}{*}{6} & \multirow{2}{*}{ Kennedy } & ALTO & 14,581 & \multirow{2}{*}{46,9} \\
\hline & & MEDIO ALTO & 3,512 & \\
\hline \multirow{2}{*}{7} & \multirow{2}{*}{ Los Mártires } & MEDIO & 2,127 & \multirow{2}{*}{50,0} \\
\hline & & MEDIO BAJO & 1,130 & \\
\hline \multirow{3}{*}{8} & \multirow{3}{*}{ Puente Aranda } & ALTO & 3,257 & \multirow{3}{*}{99,1} \\
\hline & & MEDIO & 3,663 & \\
\hline & & MEDIO ALTO & 10,228 & \\
\hline \multirow{4}{*}{9} & \multirow{4}{*}{ Rafael Uribe Uribe } & ALTO & 0,348 & \multirow{4}{*}{35,5} \\
\hline & & MEDIO & 1,623 & \\
\hline & & MEDIO ALTO & 2,355 & \\
\hline & & MEDIO BAJO & 0,584 & \\
\hline 10 & San Cristóbal & MEDIO BAJO & 0,561 & 1,1 \\
\hline 11 & Santa Fe & MEDIO BAJO & 0,028 & 0,1 \\
\hline 12 & Suba & MEDIO ALTO & 0,189 & 0,2 \\
\hline \multirow{2}{*}{13} & \multirow{2}{*}{ Teusaquillo } & MEDIO & 3,245 & \multirow{2}{*}{46,7} \\
\hline & & MEDIO ALTO & 3,381 & \\
\hline \multirow{2}{*}{14} & \multirow{2}{*}{ Tunjuelito } & $\overline{A L T O}$ & 4,813 & \multirow{2}{*}{51,9} \\
\hline & & MEDIO ALTO & 0,328 & \\
\hline
\end{tabular}

Tabla 4. Áreas de afectación por localidad. Elaboración propia. Datos propios derivados de la Secretaria de Ambiente e IDECA.

En Bogotá se cuenta con las Unidades de Planeación Zonal (UPZ), las cuales son una división administrativa desde la cual se realizan los procesos de planeación. En la tabla cinco se pueden observar las diferentes UPZ con el INCA y las áreas correspondientes de afectación encontradas. En total se analizaron 49 UPZ, de las cuales 16 se encuentran totalmente dentro

${ }^{6}$ La cual permite que se lea mejor los mapas número cuatro y cinco. 
de la zona de datos completos $100 \%$, mientras que 20 de ellas no tienen más del $50 \%$ dentro de esta zona, de las cuales 11 estuvieron por debajo del $11.5 \%$.

\begin{tabular}{|c|c|c|c|c|}
\hline ID & UPZ & INCA & $\begin{array}{l}\text { Área Afectada } \\
\text { (KM2) }\end{array}$ & $\begin{array}{c}\text { Porcentaje de Área afectada } \\
\text { por UPZ }\end{array}$ \\
\hline 1 & 20 DE JULIO & MEDIO BAJO & 0,024 & 0,91 \\
\hline 2 & AEROPUERTO EL DORADO & MEDIO ALTO & 5,408 & 72,83 \\
\hline 3 & ALAMOS & MEDIO ALTO & 2,001 & 100,00 \\
\hline \multirow{2}{*}{4} & \multirow{2}{*}{ AMERICAS } & ALTO & 3,789 & \multirow{2}{*}{100,00} \\
\hline & & MEDIO ALTO & 0,018 & \\
\hline 5 & ARBORIZADORA & ALTO & 0,295 & 9,63 \\
\hline \multirow{2}{*}{6} & \multirow{2}{*}{ BAVARIA } & ALTO & 1,520 & \multirow{2}{*}{100,00} \\
\hline & & MEDIO ALTO & 1,250 & \\
\hline 7 & BOLIVIA & MEDIO ALTO & 0,042 & 0,89 \\
\hline 8 & BOYACA REAL & MEDIO ALTO & 4,534 & 100,00 \\
\hline 9 & CALANDAIMA & ALTO & 0,145 & 4,55 \\
\hline 10 & CAPELLANIA & MEDIO ALTO & 2,719 & 100,00 \\
\hline 11 & CARVAJAL & ALTO & 4,260 & 97,22 \\
\hline \multirow{2}{*}{12} & \multirow{2}{*}{ CASTILLA } & ALTO & 3,389 & \multirow{2}{*}{86,38} \\
\hline & & MEDIO ALTO & 0,954 & \\
\hline 13 & CIUDAD JARDIN & MEDIO BAJO & 1,252 & 94,03 \\
\hline \multirow{3}{*}{14} & \multirow{3}{*}{ CIUDAD MONTES } & ALTO & 0,504 & \multirow{3}{*}{100,00} \\
\hline & & MEDIO & 0,690 & \\
\hline & & MEDIO ALTO & 3,258 & \\
\hline 15 & CIUDAD SALITRE OCCIDENTAL & MEDIO ALTO & 2,255 & 100,00 \\
\hline \multirow{2}{*}{16} & \multirow{2}{*}{ CIUDAD SALITRE ORIENTAL } & MEDIO & 0,204 & \multirow{2}{*}{100,00} \\
\hline & & MEDIO ALTO & 1,599 & \\
\hline \multirow{2}{*}{17} & \multirow{2}{*}{ DOCE DE OCTUBRE } & MEDIO & 0,018 & \multirow{2}{*}{1,77} \\
\hline & & MEDIO ALTO & 0,042 & \\
\hline 18 & ENGATIVA & MEDIO ALTO & 1,774 & 30,21 \\
\hline 19 & FONTIBON & MEDIO ALTO & 4,281 & 86,30 \\
\hline 20 & FONTIBON SAN PABLO & MEDIO ALTO & 0,220 & 6,12 \\
\hline 21 & GARCES NAVAS & MEDIO ALTO & 2,065 & 37,24 \\
\hline 22 & GRANJAS DE TECHO & MEDIO ALTO & 4,772 & 100,00 \\
\hline 23 & JARDIN BOTANICO & MEDIO ALTO & 1,616 & 100,00 \\
\hline 24 & KENNEDY CENTRAL & ALTO & 0,832 & 24,68 \\
\hline \multirow{2}{*}{25} & \multirow{2}{*}{ LA ESMERALDA } & MEDIO & 0,824 & \multirow{2}{*}{44,12} \\
\hline & & MEDIO ALTO & 0,027 & \\
\hline 26 & LA FLORESTA & MEDIO ALTO & 0,189 & 4,80 \\
\hline \multirow{2}{*}{27} & \multirow{2}{*}{ LA SABANA } & MEDIO & 0,966 & \multirow{2}{*}{29,69} \\
\hline & & MEDIO BAJO & 0,371 & \\
\hline 28 & LAS CRUCES & MEDIO BAJO & 0,028 & 3,00 \\
\hline \multirow{2}{*}{29} & \multirow{2}{*}{ LAS FERIAS } & MEDIO & 0,000 & 7005 \\
\hline & & MEDIO ALTO & 3,313 & 10,03 \\
\hline 30 & MAPCO FIDEI SUA PEZ & MEDIO & 0,077 & 1025 \\
\hline 30 & 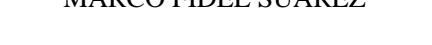 & MEDIO ALTO & 0,112 & \\
\hline
\end{tabular}


Revista de Geografia (Recife) V. 34, No. 1, 2017

\begin{tabular}{|c|c|c|c|c|}
\hline 31 & MINUTO DE DIOS & MEDIO ALTO & 2,242 & 60,11 \\
\hline 32 & MODELIA & MEDIO ALTO & 2,614 & 100,00 \\
\hline \multirow{2}{*}{33} & \multirow{2}{*}{ MUZU } & ALTO & 1,893 & \multirow{2}{*}{100,00} \\
\hline & & MEDIO ALTO & 0,633 & \\
\hline \multirow{2}{*}{34} & \multirow{2}{*}{ PARQUE SALITRE } & MEDIO & 0,320 & \multirow{2}{*}{34,08} \\
\hline & & MEDIO ALTO & 0,243 & \\
\hline \multirow{2}{*}{35} & \multirow{2}{*}{ PARQUE SIMON BOLIVAR - CAN } & MEDIO & 1,186 & \multirow{2}{*}{72,27} \\
\hline & & MEDIO ALTO & 1,693 & \\
\hline \multirow{2}{*}{36} & \multirow{2}{*}{ PUENTE ARANDA } & MEDIO & 0,352 & \multirow{2}{*}{100,00} \\
\hline & & MEDIO ALTO & 3,210 & \\
\hline \multirow{2}{*}{37} & \multirow{2}{*}{ QUINTA PAREDES } & MEDIO & 1,031 & \multirow{2}{*}{62,84} \\
\hline & & MEDIO ALTO & 0,061 & \\
\hline \multirow{4}{*}{38} & \multirow{4}{*}{ QUIROGA } & ALTO & 0,348 & \multirow{4}{*}{97,58} \\
\hline & & MEDIO & 1,105 & \\
\hline & & MEDIO ALTO & 2,244 & \\
\hline & & MEDIO BAJO & 0,005 & \\
\hline \multirow{4}{*}{39} & \multirow{4}{*}{ RESTREPO } & ALTO & 0,144 & \multirow{4}{*}{100,00} \\
\hline & & MEDIO & 1,316 & \\
\hline & & MEDIO ALTO & 1,076 & \\
\hline & & MEDIO BAJO & 1,008 & \\
\hline \multirow{2}{*}{40} & \multirow{2}{*}{ SAN JOSE } & MEDIO & 0,441 & \multirow{2}{*}{49,13} \\
\hline & & MEDIO BAJO & 0,580 & \\
\hline \multirow{2}{*}{41} & \multirow{2}{*}{ SAN RAFAEL } & ALTO & 0,860 & \multirow{2}{*}{100,00} \\
\hline & & MEDIO ALTO & 2,430 & \\
\hline 42 & SANTA CECILIA & MEDIO ALTO & 3,083 & 100,00 \\
\hline \multirow{2}{*}{43} & \multirow{2}{*}{ SANTA ISABEL } & MEDIO & 1,160 & $0 \approx 0$ \\
\hline & & MEDIO BAJO & 0,759 & 90,00 \\
\hline 44 & SOSIEGO & MEDIO BAJO & 0,537 & 22,87 \\
\hline 45 & TIMIZA & ALTO & 0,475 & 11,03 \\
\hline 16 & TINT & ALTO & 0,172 & 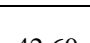 \\
\hline 46 & IINI AL NORIE & MEDIO ALTO & 1,290 & 42,60 \\
\hline 17 & УГNГCL & ALTO & 4,813 & 7751 \\
\hline 47 & VENECIA & MEDIO ALTO & 0,328 & $1 /, 51$ \\
\hline 48 & ZONA FRANCA & MEDIO ALTO & 0,396 & 8,06 \\
\hline 10 & $7 O N A$ INDUCTDII & MEDIO & 2,621 & 0560 \\
\hline 49 & ZUNA INDUDIKIAL & MEDIO ALTO & 0,696 & 95,69 \\
\hline
\end{tabular}

Tabla 5. Área total afectada por Unidades de Planeación Zonal (UPZ)

El mapa número cinco muestra la distribución de áreas por UPZ por Bogotá. Hay UPZ en las cuales el INCA es Alto: Calandaima, Carvajal, Kenney Central y Timiza dentro del área total analizada, mientras que Américas, Bavaría, Castilla, Muzu, San Rafael, Tintal Norte y Venecia tienen sectores con Contaminación Alta. 20 de Julio, Ciudad Jardín, Las Cruces y Sosiego tiene un INCA Medio Bajo dentro de la zona de datos completos. Santa Isabel, San Jose y La Sabana tiene partes con este indicador e INCA Medio. Mientras que las UPZ 
Ciudad Montes contiene los tres indicadores superiores y Quiroga y Restrepo tienen los cuatro INCA dentro de su área.

INCA por Unidades de Planeación Zonal en Bogotá D.C. para el 2015

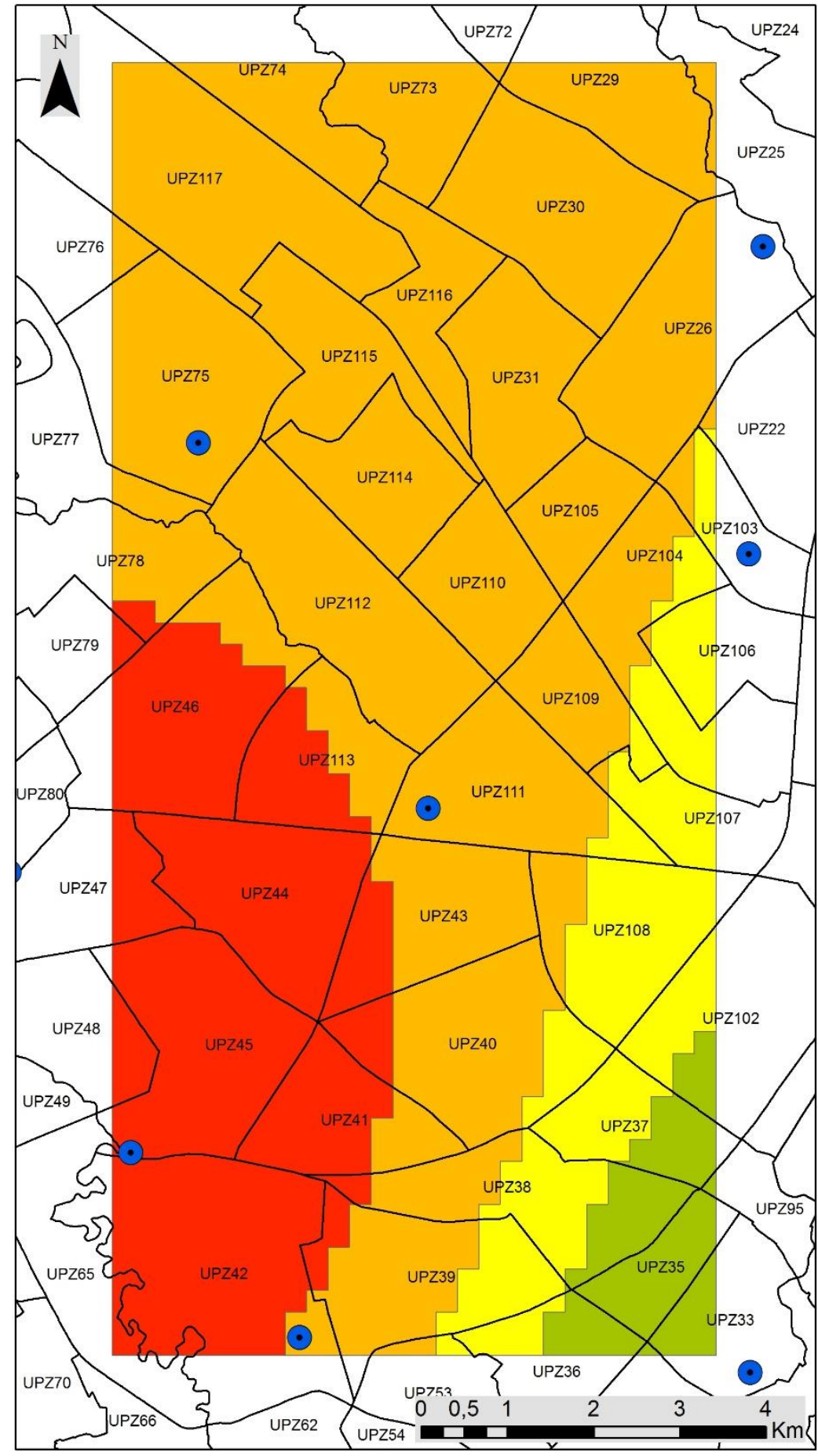

\begin{tabular}{|c|c|}
\hline Código & Nom bre \\
\hline UPZ34 & 20 DE JULIO \\
\hline UPZ117 & AEROPUERTO EI DORADO \\
\hline UPZ116 & ALAMOS \\
\hline UPZ44 & AMERICAS \\
\hline UPZ65 & ARBORIZADORA \\
\hline UPZ113 & BAVARIA \\
\hline UPZ72 & BOLIVIA \\
\hline UPZ30 & BOYACA REAL \\
\hline UPZ79 & CALANDAIMA \\
\hline UPZ115 & CAPELLANIA \\
\hline UPZ45 & CARVAJAL \\
\hline UPZ46 & CASTILLA \\
\hline UPZ35 & CUUDAD JARDIN \\
\hline UPZ40 & CIUDAD MONTES \\
\hline UPZ110 & GUDAD SALIRE OCCIDENTAL \\
\hline UPZ109 & CIUDAD SALITRE ORIENTAL \\
\hline UPZ22 & DOCE DE OCTUBRE \\
\hline UPZ74 & ENGATIVA \\
\hline UPZ75 & FONTIBON \\
\hline UPZ76 & FONTIBON SAN PABLO \\
\hline UPZ73 & GARCES NAVAS \\
\hline UPZ112 & GRANJAS DE TECHO \\
\hline UPZ105 & JARDIN BOTANICO \\
\hline UPZ47 & KENNEDY CENTRAL \\
\hline UPZ106 & LA ESMERALDA \\
\hline UPZ25 & LA FLORESTA \\
\hline UPZ102 & LA SABANA \\
\hline UPZ95 & LAS CRUCES \\
\hline UPZ26 & LAS FERAS \\
\hline UPZ53 & MARCO FIDE SUAREZ \\
\hline UPZ29 & MINUTO DE DIOS \\
\hline UPZ114 & MODELIA \\
\hline UPZ41 & MUZU \\
\hline UPZ103 & PARQUE SALTRE \\
\hline UPZ104 & PARQUE SIMON BOLIVAR - CAN \\
\hline UPZ111 & PUENTEARANDA \\
\hline UPZ107 & QUINTA PAREDES \\
\hline UPZ39 & QUIROGA \\
\hline UPZ38 & RESTREPO \\
\hline UPZ36 & SANJOSE \\
\hline UPZ43 & SANRAFAE \\
\hline UPZ31 & SANTA CECILIA \\
\hline UPZ37 & SANTA ISABE \\
\hline UPZ33 & SOSIEGO \\
\hline UPZ48 & TIMZA \\
\hline UPZ78 & TINTAL NORTE \\
\hline UPZ42 & VENECIA \\
\hline UPZ77 & ZONA FRANCA \\
\hline UPZ108 & ZONA INDUSTRAAL \\
\hline \multicolumn{2}{|c|}{ Leyenda } \\
\hline \multicolumn{2}{|r|}{ Red de Estaciones Calidad de Aire } \\
\hline & \multirow{2}{*}{ ] Unidades de Planeación Zonal } \\
\hline INCA & \\
\hline \multicolumn{2}{|r|}{ MEDIO BAJO } \\
\hline \multicolumn{2}{|r|}{ MEDIO } \\
\hline \multicolumn{2}{|r|}{ MEDIO ALTO } \\
\hline & ALTO \\
\hline
\end{tabular}

Mapa 5. INCA por Unidades de Planeación Zonal en Bogotá para el 2015. Fuente de Información y Espaciales: Secretaría de Ambiente Distrito Capital Infraestructura de Datos Espaciales para el Distrito Capital (IDECA). Información propia Derivada de Geoprocesamientos. Elaboración Propia 
En esa medida, aquellas UPZ que contienen dentro el INCA Alto y Medio Alto debería tener planes de manejo integral de los contaminantes, para así desarrollar procesos que permitan la mitigación de efectos negativos a sus pobladores. En especial a aquellos que se encuentran más cercanos a zonas de contaminación alta y que corresponden a las localidades de Kennedy, Tunjuelito y Ciudad Bolívar.

Si se tuvieran datos que permitiera analizar completamente las localidades que se encuentran con este tipo de INCA Alto se podría hacer planes más eficientes para las UPZ y las zonas en las cuales se pueda visibilizar más la afectación, así como las zonas en donde se producen los contaminantes, pues lo que se muestra es la dispersión y no el lugar en donde se producen.

La calidad de aire para las diferentes localidades y UPZ a través de este ejercicio muestran que hay sectores en donde se concentra los contaminantes y que son necesarios analizar, pero falta establecer si hay relación entre la dispersión y las corrientes de aire, para ello se realizó el tratamiento de datos correspondiente para generar el mapa de flujos de las corrientes en promedio anual.

El tratamiento de datos de viento permite conocer la forma en que se dispersa algunos de los contaminantes en flujos, para esto se realiza una interpolación en donde se tienen en cuenta las variables de dirección y velocidad, los cuales deben asumirse desde la composición del vector de descomposición ortogonal, así como las variables obtenidas y los previos que se puedan dar (GONZÁLEZ FERREIRO e BOSQUES SENDRA, 2008) al traspasar la información de una manera raster a vector.

El mapa cinco muestra la velocidad y dirección del viento, allí se puede ver como aumenta el INCA a medida que el flujo de viento se dirige al occidente en la zona de datos completos. Por lo tanto, puede existir una correlación entre las corrientes de aire que se dan en Bogotá y la dinámica de los contaminantes que se dispersan por aire. En este caso, la estación de Fontibón permite conocer mejor la relación del flujo que se da dentro de la ciudad. 


\section{Promedio de Velocidad y Dirección del Viento para Bogotá en el 2015 en el Área de Medición de Calidad de Aire}

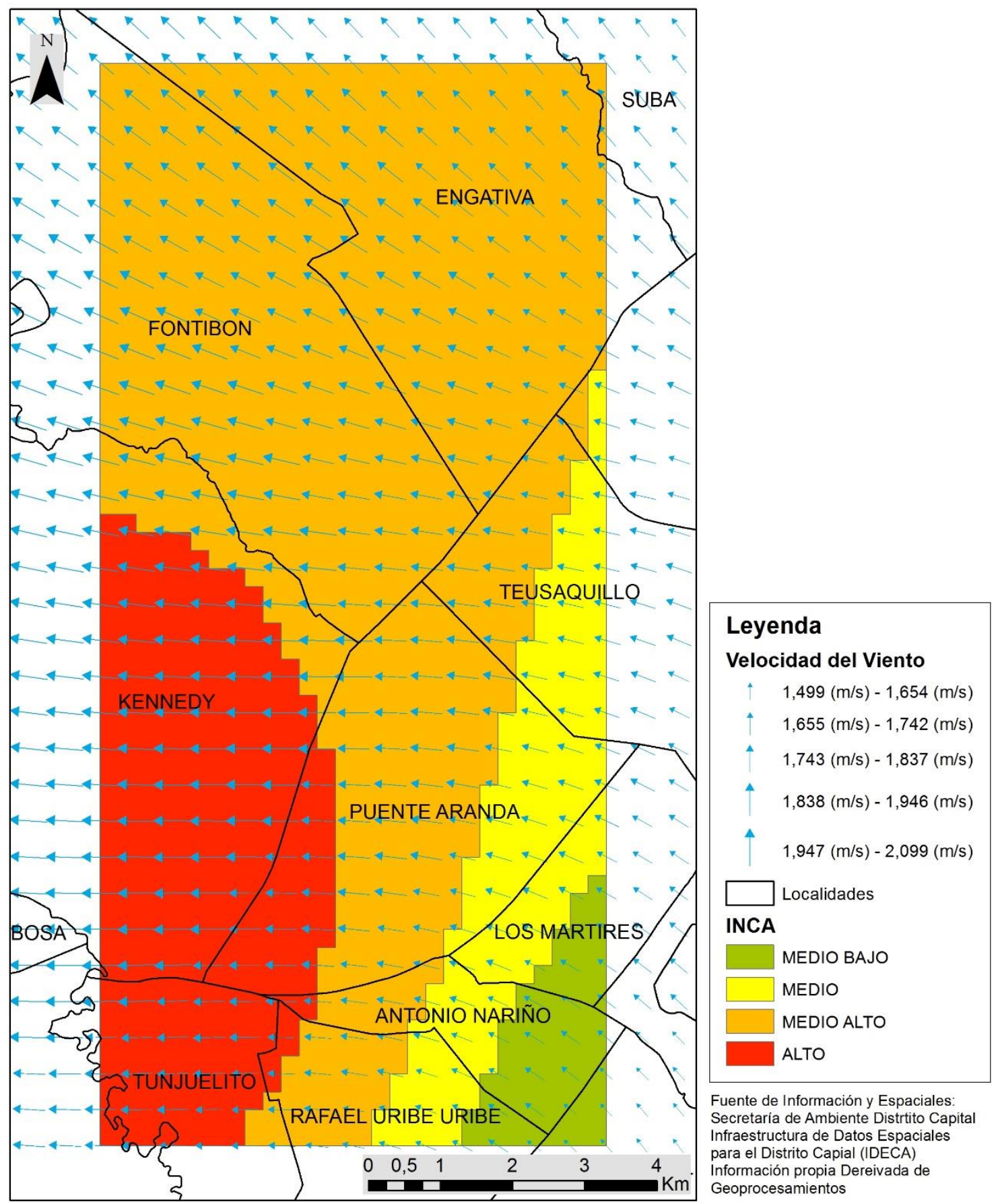

Mapa 6. Promedio de velocidad y dirección del viento para Bogotá en el 2015 en el área de Medición de calidad de Aire. Elaboración Propia.

Como Menciona Galarza (2010) es necesario que se generen mejores tomas de muestras que permitan conocer las emisiones que se producen en la zona estudiada, teniendo 
en cuenta que la adquisición de la información es compleja y que en muchos casos no se puede tener acceso a ella, o en otros casos no hay elementos que permitan la toma de muestras de esas zonas. Por lo tanto, es necesario que se generen mecanismos que permitan conocer el impacto de las emisiones generadas dentro de áreas urbanas que lastimosamente aquí solo representan una pequeña franja del total de la ciudad.

Como menciona Cañada, Vidal y Moreno "a pesar de los esfuerzos que se han venido realizando para mejorar la calidad del aire, la contaminación atmosférica sigue implicando un elevado riesgo ambiental, humano y urbano, que tiene que ser atajado con una perspectiva integral e integradora" (2011, p. 120), más cuando no se cuenta con el total de información que permita relacionar e identificar los contaminantes de aire, lo que condiciona la elaboración del proceso de análisis de los datos cartográficos.

Asimismo existen dificultades que no permiten correlacionar los datos, como lo menciona Colsa et. Al. (2014) Entre estos se encuentra la cantidad de puntos con los cuales se toma la información, condicionando las interpolaciones que se efectúan y que, al tener vacíos en la captura del dato, se modifica y la representación cartográfica tiene efectos de representación. Opazo (2012) encuentra que es necesario tener una cantidad de estaciones que permitan el análisis en áreas más pequeñas, y con ello acceder a información más confiable ${ }^{7}$.

Es necesario contar con los datos locales de las estaciones que miden los valores, pero es importante que estás generen una información continua y que no puede desligarse de las necesidad del software, puesto que esto permite la configuración del modelo a realizar (ASTUDILLO ROMERO, 2012), el cual he intentado que capte la relaciones que se dan entre la zona en la cual se pude medir esta información.

Esto incluye los datos con los cuales no se pudo contar, para así generar un modelo mayor que permita establecer las correlaciones sobre diversos departamentos, manejando un intervalo de confiablidad más alto. Es necesario que se continúe con este tipo de investigaciones, para así poder encontrar las fallas que se generan en la adquisición de la información. Las variables aquí presentadas tienen vacíos y que son necesarios para entender otros aspectos, como por ejemplo la justicia ambiental, en donde se establecen relaciones reciprocas entre las necesidades de la población y el ambiente; y que en el caso de contaminantes atmosféricos son un imperativo (MORENO JIMÉNEZ e CAÑADA TORRECILLA, 2007)

\footnotetext{
${ }^{7}$ Claro que el autor hace referencia al material particulado correspondiente a la ciudad de Santiago, así como la temperatura como determinante en el cambio de la dispersión de ese material.

$\begin{array}{lcc}\text { Aguilar Galindo, } 2017 & \text { ISSN 0104-5490 }\end{array}$
}




\section{CONCLUSIONES}

En el desarrollo de la investigación de la Calidad de Aire para Bogotá en el 2015 se comprobó que varias de las estaciones de la Red de Monitoreo de Calidad e Aire no miden en igualdad de condiciones los contaminantes emitidos por diferentes fuentes, lo cual produce que no se puedan hacer análisis del área total del área de la ciudad.

Con las ausencias de información se pudo lograr analizar un área total de 105 KM2 en la cual se determinó un Indicador de Calidad por Área (INCA) lo que facilito el análisis de los datos agrupados por contaminantes, esto gracias a la realización de un Sistema de Información Geográfico (SIG) que dio como resultado las áreas de mayor concentración de contaminantes que afectan la calidad del aire por dispersión.

Se detectó dentro del SIG que hay una zona con datos completos que pudo ser analizada y en la cual se toma la relación de los datos como cierta (confiable) y que permite entrar a discutir el grado de dispersión de los contaminantes por el INCA. En esta zona se evidencio que cinco UPZ tienen una condición alta de concentración por dispersión de contaminantes, mientras que nueve de ellas presentan áreas con este indicador.

También se encontró que las corrientes de aire tienen el mismo patrón de comportamiento que los INCA, mostrando como la dirección del viento se encuentra en disposición del menor hacia el mayor, haciendo que estos se encuentren en las zonas de mayor concentración.

\section{RECOMENDACIONES}

Se es necesario que se desarrolle un muestreo eficiente, en el cual no aparezcan vacíos de información y que permita evidenciar las emisiones que ocurren en cada una de las localidades.

Al mismo tiempo es necesario que se mejore la red de medición del aire, pues hay zonas que no se cubren y las cuales producen una gran cantidad de gases y material particulado, como lo son el Relleno Sanitario de Doñas Juana, el parque minero el Mochuelo (ubicadas en la Localidad de Ciudad Bolívar) y el parque minero de Usme.

Es necesario que el ejercicio se realice de manera diaria, semanal y mensual con un sistema que cartografíe rápidamente los contaminantes, para así establecer las zonas que 
necesitan mitigación o control de los efectos nocivos que esos contaminantes producen, los cuales no se han podido evidenciar por la falta de información con la que cuenta el distrito.

\section{BIBLIOGRAFÍA}

ASTUDILLO ROMERO, M. MODELACIÓN DE DISPERSIÓN ESPACIAL DE CONTAMINANTES DEL AIRE EN LA CIUDAD DE CUENCA. UNIVERSIDAD SAN FRANCISCO DE QUITO. QUITO. 2012.

BELTRÁn CALVO, A. Efectos de Contaminación del aire en la salud. Una perspectiva de justicia ambiental para la ciudad de Bogotá. Universidad de los Andes. Bogotá. 2013.

CAÑADA TORRECILLA, M. R.; MORENO JIMÉNEZ, A.; GONZÁLEZ LORENZO, H. MODELADO DE LA CALIDAD DEL AIRE URBANO. UN EXPERIMENTO METODOLÓGICO CON TÉCNICAS DE INTERPOLACIÓN ESPACIAL. Boletín de la A.G.E., n. 65, p. 317-342, 2014. ISSN ISSN 0212-9426.

CAÑADA TORRECILLA, R.; VIDAL DOMÍNGUEZ, M. J.; MORENO JIMÉNEZ, A. Interpolación espacial y visualización cartográfica para el análisis de la justicia ambiental: ensayo metodológico sobre la contaminación por partículas atmosféricas en Madrid. GeoFocus, n. 11, p. 118-154, 2011. ISSN ISSN 1578-5157.

CAÑADA TORRENCILLA, R.; MORENO JIMÉNEZ, A. Monitoreo con SIG de la calidad de la atmósfera urbana para la gobernanza local: el caso de Madrid. Revista Ciencias Espaciales, v. 8, n. 2, p. 431-451, 2015.

COLSA PÉREZA, A. et al. Caracterización de la calidad del aire en la ciudad de Valencia: Un análisis basado en la interpolación espacial de contaminantes. XVI Congreso Nacional de Tecnologías de la Información Geográfica. Alicante: [s.n.]. 2014. p. 1-11.

ELJAUDE, L. Determinación de áreas de isocontaminación en la zonaurbana de Santa Fe de Bogotá DC mediante la utilización de líquenes. Universidad de la Salle. Bogotá. 1995.

FERNÁNDEZ GARCÍA, F. Contaminación atmosférica y calidad del aire en Madrid: análisis de las concentraciones de SO2, CO, NO2, OZONO y PM10 (1980-2003). Estudios Geográficos, v. 64, n. 259, p. 507-532, 2005. ISSN ISSN 0014-1496.

GALARZA RODRIGO, J. D. J. Desarrollo de una metodología sistematizada basada en SIG 2D y los factoresd de emisión para evaluar las fuentes de emisiones al aire. Universidad de Guayaquil. Guayaquil. 2010.

GARCÍA ÁVILA, P. A.; ROJAR, N. Y. Análisis del origen PM10 y PM2.5 en Bogotá usando gráficos polares. Mutis, v. 6, n. 2, p. 47-58, 2016.

GONZÁLEZ FERREIRO, D.; BOSQUES SENDRA, J. Generating a map of wind in a GIS. Boletín de la A.G.E., n. 47, p. 369-370, 2008. 
HAWKSWORTH, D.; ITURRIAGA, T.; CRESPO, A. Líquenes como bioindicadores inmediatos de contaminación y cambios medio-ambientales en los trópico. Rev. Iberoam. Mic., n. 22, p. 72-82, 2005.

LONDOÑO, J.; CORREA, M. A.; PALACIO, C. A. Estimación de las emisiones de contaminantes atmosféricos provenientes de fuentes móviles en el área urbana de Envigado, Colombia. Revista de la Escuela de Ingeniería de Antioquía, n. 16, p. 149-162, 2011. ISSN ISSN 1794-1237.

MENA, R. Calidad de aire en Quibdó mediante bioindicadores. Revista Bioetnia, v. 9, n. 2, p. 215-227, 2012. ISSN ISSN 1990-0561.

MINISTERIO DE AMBIENTE, V. Y. D. T. Manual de operaciones de Sistema de Vigialancia de la Calidad del Aire. Bogotá: Ministerio de Ambiente, Vivienda y Desarrollo Territorial, 2010.

MINISTERIO DE AMBIENTE, V. Y. D. T. Resolución 0610 de 2010. Bogotá D.C. 2010.

MORENO JIMÉNEZ, A.; CAÑADA TORRECILLA, R. Justicia ambiental y contaminación atmosférica por dióxido de azufre en Madrid: Análisis espacio-temporal y valoración con sistemas de información geográfica. Boletin de la A.G.E., n. 44, p. 301-324, 2007.

MOSCOSO VANEGAS, D. L.; ASTUDILLO ALEMÁN, A. L.; VÁZQUEZ FREIRE, V. E. Modelamiento de la calidad del aire en la ciudad de Cuenca-Ecuador. ITECKNE, v. 12, n. 2, p. 188-197, 2015. ISSN ISSN 1692 - 1798.

OPAZO ALBARRÁN, D. Distribución espacial de la contaminación por material particulado en Santiago y su relación con las temperaturas del aire y los vientros para el año 2009. Anales Sociedad Chilena de Ciencias Geográficas, p. 27-33, 2012. ISSN ISSN0717-3946.

POSADA PARRA, M. L. Construcción de una aplicación WEB de mapas, para visualización de información relacionada con calidad de aire en la ciudad de Bogotá, utilizando software libre y de codigo abierto. Universidad Militar Nueva Granada. Bogotá D.C. 2015.

QUICO BERRIO, L. Elaboración de mapas en SIG sobre información de calidad ambiental. Lima: Ministerio del Ambiente de Perú, 2012. Informe Final.

RODRÍGUEZ RODRÍGUEZ, H. M. F. Apliación de métodos de interpolación y modelamiento geoestadístico en la evaluación de la calidad e aire en Bogotá D.C. Universidad Militar Nueva Granada. Bogotá D.C. 2014.

ROMERO, H.; OPAZO, D. Ecología política de los espacios urbanos metropolitanos: geografía de la injusticia ambiental. Revista Geográfica de Amércia Central, n. Especial EGAL, p. 1-16, 2011. ISSN SSN 2115-2563.

SECRETARÍA DE AMBIENTE DE BOGOTÁ. Red Monitoreo de Calidad de Aire de Bogotá, 15 Abril 2016. Disponivel em: 〈http://201.245.192.252:81>. 